ISSN electrónico: 2172-9077

https://doi.org/10.14201/fjc2017155773

\title{
LOS JÓVENES UNIVERSITARIOS Y SUS PAUTAS DE CONSUMO Y DIFUSIÓN DE NOTICIAS SEGÚN LA TENDENCIA IDEOLÓGICA
}

\section{Young University Students and their Patterns of Consumption and Dissemination of News According to the Ideological Trend}

Dra. Beatriz CATALINA-GARCÍA

Profesora Visitante. Universidad Rey Juan Carlos, España

beatriz.catalina@urjc.es

iD http://orcid.org/0000-0003-0464-3225

Dr. Manuel MONTES VOZMEDIANO

Profesor Titular Interino. Universidad Rey Juan Carlos, España manueljavier.montes@urjc.es

(iD http://orcid.org/0000-0001-6624-8699

Dr. Antonio GARCÍA JIMÉNEZ

Catedrático de Periodismo. Universidad Rey Juan Carlos, España

antonio.garcia@urjc.es

http://orcid.org/0000-0002-8423-9486

Fecha de recepción del artículo: 04/09/2017

Fecha de aceptación definitiva: 15/10/2017

\begin{abstract}
RESUMEN
Este texto analiza los resultados de una encuesta exploratoria entre estudiantes de la Universidad Rey Juan Carlos con el objeto de analizar en qué medida y de qué forma el posicionamiento político influye en el consumo y acceso a las noticias, con especial interés en el plano digital. Esta perspectiva se establece en relación a aspectos como los medios consultados, la credibilidad otorgada o el modo en que se comparte la información de actualidad. A tal efecto, se ha trabajado sobre la base de una encuesta autoadministrada durante un mes a 297 alumnos de Comunicación. Se ha podido observar una correlación entre la ideología y la mayor atención en Internet, junto a la relevancia de la televisión convencional en combinación con el acceso a las noticias digitales. En este contexto, son los medios sociales, antes que la prensa, el espacio más habitual de acceso a la información de actualidad. También se confirma la idea de credibilidad asociada a la prensa y radio tradicionales.
\end{abstract}

Palabras clave: jóvenes; consumo de noticias; internet; redes sociales; credibilidad; ideología.

\begin{abstract}
This paper aims to analyze to what extent and the ways in which the political positions of Spanish young people has influence on the consumption and access to news, with special interest in the digital point of view. This perspective is established in relation to aspects such as the media consulted, the credibility and the forms in which information is shared. In this sense, a survey self-administered for one month among 297 Communication students is conducted. It has been possible to observe a correlation between the ideology and the greater attention in Internet, in addition to the relevance of the conventional television in combination with the access digital news. In this context, social media become a most commonly space for accessing digital news instead of digital newspapers. This paper also confirms the idea of credibility associated to both traditional press and radio.
\end{abstract}

Key words: young people; consumption; news; internet; social networks; credibility; ideology. 


\section{INTRODUCCIÓN}

Los hábitos de consumo de la información de actualidad por parte de los jóvenes, así como la constatación de su creciente papel como prosumidores configuran una realidad cambiante de incuestionable interés social que suscita el estudio desde diversos prismas.

Este texto sitúa el punto de mira en la tendencia ideológica de los universitarios españoles con el fin de determinar en qué medida esta condiciona sus pautas de consulta y difusión de las noticias. Se estudia si la tendencia ideológica, desde el punto de vista de sus convicciones políticas, se relaciona con la pluralidad de los medios que consulta, la credibilidad que les otorga, el tipo de información que les interesa o el modo en que comparte o distribuye la información de actualidad: a quiénes, a través de qué canales o qué fuentes emplea, entre otras consideraciones.

\section{ESTADO DE LA CUESTIÓN}

El interés de los jóvenes por la información de actualidad, cuya consulta se ha trasladado de los soportes en papel a los nuevos formatos y de los medios digitales a redes sociales como Facebook o Twitter (Catalina-García, García y Montes, 2015), está en sintonía con una elevada preocupación cívica por parte de este colectivo (Casero-Ripollés, 2012). Al ser creciente su papel como prosumidores, nos interesa conocer qué influencia tienen factores como la ideología en la consulta y divulgación de la información. El análisis de casos como el uso de Twitter durante las elecciones de mayo del 2015 en España (Said-Hung, Prati y Cancino-Borbón, 2017) nos permite entender el empleo que se da a las redes sociales. En esta ocasión Twitter se utilizó mayoritariamente como mecanismo de divulgación de los acontecimientos, si bien cuando los mensajes dejaban patente una clara orientación ideológica, abundaban los mensajes con una tendencia progresista o de izquierdas.

Otros estudios han vinculado la ideología de los jóvenes universitarios con pautas de organización social, relacionando los valores de bienestar de los demás a jóvenes con posicionamiento ideológico de izquierdas y con formación en carreras humanísticas, mientras que los jóvenes de derechas se alineaban con tendencias de dominancia social y optaban por estudios tradicionales y técnicos que enfatizaban metas como la consecución del poder (Zubieta, et al., 2008). Unas conclusiones en la línea de las categorizaciones expuestas por Espinoza y Calderón (2006) en las que la ideología de izquierdas se asocia a valores de cambio y la de derechas a la tradición y conformidad.

Cabe destacar el papel ascendente de la red social Twitter, no tanto como vehículo directo de consulta de la información de actualidad, pero sí como canal indirecto de acceso a la misma dado su potencial para distribuir acontecimientos noticiosos (Kim, et al., 2014); en paralelo, Bernal (2015) observa que la generación de redes sociales ha supuesto para el público la posibilidad de cambiar una agenda única que aportan los medios de comunicación convencionales a una agenda múltiple activa, abierta y colaborativa. Un hecho que se alinea con las conclusiones extraídas por Qayyum et al. (2010) si bien estos investigadores consideran el consumo de información en Internet no como una actividad de primer orden, sino como una consecuencia secundaria de la actividad de navegación. Los jóvenes tienen otros objetivos prioritarios en su actividad en la Red como el de la interacción social (Carlsson, 2011) siendo la consulta de la actualidad una deriva accidental (Patterson, 2007). En todo caso, los usuarios valoran positivamente que los medios digitales favorezcan, mediante conexiones con otros recursos online, que las informaciones se puedan contrastar o ampliar, así como la inclusión de espacios que propicien la interactividad (Gutiérrez-Coba, et al., 2012).

Cuando el interés de los ciudadanos por la política -y otros temas de actualidad informativa- decae, el modo en que los medios de comunicación procuran revertir la situación depende del contexto 
histórico y social de cada nación, si bien se aprecia una tendencia global hacia el modelo del infoentretenimiento en el producto informativo. Dentro de esta categorización coexisten diversas variantes: los temas políticos se tratan de un modo sensacionalista (Hanusch, 2013) y las esferas privadas de los protagonistas se privilegian (Stanyer, 2012) como consecuencia de la denominada era digital (CaseroRipollés, Ortells-Badenes y Rosique-Cedillo, 2014). Existe pues una tendencia a priorizar el estilo ameno de la información, con independencia de las audiencias de los medios, ya sean populares o elitistas (Mellado-Ruiz, López-Rabadán y Elortegui, 2016).

Con independencia del contenido consultado queda patente la tendencia de los jóvenes por compartir aquello que consideran interesante (Jiménez y Gaitán Caballero, 2013), siendo Facebook y Twitter las redes preferidas. A propósito de esta última vía, López-Meri (2016) observa que en dos hashtags que marcaron viralidad en Twitter (\#Arde-Valencia y \#25N, relacionados respectivamente con los incendios forestales ocurridos en Valencia en el 2012 y con las elecciones en Cataluña del mismo año), los ciudadanos tienden a divulgar y promocionar sus contenidos. En este estudio analizaremos si la ideología también condiciona los canales, el modo en que se comparte, así como el contenido que se difunde. De este modo las conclusiones extraídas serán complementarias a las obtenidas en investigaciones como la de Gómez Rubio y López Vidales (2016) que muestra cómo las cadenas televisivas, en canales sociales como Twitter, priorizan de forma más evidente que en la emisión convencional, la publicación de ciertas noticias en función de su ideología.

Finalmente, esta investigación se deriva de diversas bases teóricas y prácticas. Por un lado, de los estudios como el de Rayén, Bachmann y Mujica (2014) que se adentra en el consumo de noticias por parte de colectivos adolescentes y jóvenes en Chile. Por otro, los trabajos orientados a conocer los usos de Internet y redes sociales, en diversos espacios (Pérez Tornero, et al., 2015). También son de interés aquellos que se aproximan a cuestiones relevantes a la hora del consumo de noticias, en concreto de la credibilidad (García Avilés, et al., 2014), así como las investigaciones centradas en el comportamiento digital de los jóvenes en el marco de movilizaciones sociales (Valenzuela, et al., 2012) o en las que se observa la polarización a partir de la exposición a las redes sociales (Tewksbury y Riles, 2015).

\section{OBJETIVOS}

El objetivo general de este trabajo es detectar las tendencias que tienen los universitarios españoles, en su calidad de ciudadanos, para informarse de la actualidad. Se pretende averiguar si el hábito que desarrollan en torno a la consulta de noticias varía en función de su auto-posicionamiento ideológico, cuya base se entiende en la tradicional categorización Izquierda-Derecha que se otorga y autootorgan los distintos grupos políticos de buena parte de los Estados democráticos mundiales. Esta visión diádica, presente desde la Revolución Francesa, tiene como función, según Bobbio (1995, p. 94), «dar solo un nombre a la persistente, y persistente por esencial, composición dicotómica del universo político».

Para conocer más profunda y detalladamente la tendencia ideológica de los encuestados en este estudio, se utiliza una escala de Likert con valores entre el 1 y el 7 que posibilitan un análisis operacional de los resultados obtenidos en los siguientes ítems: Extrema-Izquierda, Izquierda, CentroIzquierda, Centro, Centro-Derecha, Derecha y Extrema Derecha.

A partir de esta premisa, se plantea alcanzar los siguientes objetivos particulares:

O1. Establecer la frecuencia, los motivos, así como los medios y modos que utilizan los distintos grupos establecidos para la consulta y contraste de la información de actualidad que les llega desde diferentes vías. 
O2. Comprobar si los jóvenes encuestados se convierten en fuentes de información con la difusión a su entorno de las noticias que reciben de los distintos medios y formatos que consultan. Plantear si la frecuencia de este cometido, así como el tipo de medios que utilizan para ello, guarda relación con sus convicciones políticas.

O3. Conocer la percepción que estos universitarios tienen de las distintas vías que eligen para conocer la información y observar si existen diferencias, en cuanto a su auto-posicionamiento ideológico, sobre el grado de confianza (credibilidad) que depositan en estos recursos y el nivel de imparcialidad que les otorgan.

\section{Metodología}

La presente investigación toma como base la encuesta de carácter exploratorio realizada a 297 alumnos matriculados en diferentes grados de la Facultad de Ciencias de la Comunicación de la Universidad Rey Juan Carlos (Madrid-España), de titularidad pública. Con las respuestas obtenidas se elaboró una base de datos que fue tratada posteriormente con el programa estadístico SPSS Statistics 18.0. para establecer relaciones de contingencia en función de la tendencia ideológica manifestada por los encuestados.

Las edades de los sujetos oscilan entre los 18 y los 30 años, con una media resultante de 20,94 años y una moda de 21. Los estudiantes autocumplimentaron el cuestionario en las aulas. Del total de encuestados, se registra un 5,1\% de resultados perdidos que corresponde a jóvenes que no indicaron su ideología. En el resto de los que sí señalaron su posición, 282, hay una clara inclinación hacia la izquierda en distintos grados y, en particular, hacia el Centro-Izquierda (36,2\%). Un tercio de los encuestados indica una tendencia hacia el Centro; y en los polos radicales, solo uno señala la ExtremaIzquierda y ninguno la Extrema-Derecha (Tabla I).

Tabla I. Posición política de los encuestados

\begin{tabular}{|cc|c|c|c|c|}
\hline & & & & Porcentaje \\
Válidos & Extrema Izquierda & 1 &, 3 &, 4 &, 4 \\
& Izquierda & 39 & 13,1 & 13,8 & 14,2 \\
& Centro-Izquierda & 102 & 34,3 & 36,2 & 50,4 \\
& Centro & 95 & 32,0 & 33,7 & 84,0 \\
& Centro-Derecha & 35 & 11,8 & 12,4 & 96,5 \\
& Derecha & 10 & 3,4 & 3,5 & 100,0 \\
& Extrema-Derecha & 0 &, 0 &, 0 & 100,0 \\
& Total & 282 & 94,9 & 100,0 & \\
Perdidos & 15 & 5,1 & & \\
& Sistema & 297 & 100,0 & & \\
\hline
\end{tabular}

Fuente: elaboración propia.

La encuesta consta de 20 preguntas, 7 de ellas con carácter de respuesta múltiple y 2 que sirvieron de filtro. Las relacionadas con la percepción de la imparcialidad de los medios y la credibilidad que mantienen hacia ellos se han elaborado bajo una escala de Likert cuyos valores oscilan entre 1 y 5 , siendo el parámetro mínimo nada creíble/imparcial; y el máximo totalmente creíble/imparcial. 
El tiempo requerido para completar el cuestionario oscila entre los 5 y los 10 minutos. Al margen de las primeras indagaciones referidas al perfil de los encuestados, de su tendencia ideológica y de su participación social, el resto del cuestionario se divide en los siguientes ejes temáticos:

- Consulta de información. Se planteaba la frecuencia diaria (entre nunca y tres o más veces al día); los motivos que les induce a informarse; contenidos que más les interesa y las vías más recurrentes para esta práctica. En este último aspecto se les pregunta tanto por los medios de comunicación dedicados principalmente a difundir noticias (televisión, radio y prensa en sus distintas versiones online y tradicionales), como por otras formas digitales que, aunque su cometido es diverso, también transmiten información de actualidad; en este caso se incluyen redes sociales, blogs, servicios de mensajería, buscadores y otras formas de naturaleza indeterminada. Las respuestas correspondientes a los distintos grupos de ítems fueron sumadas y se obtuvo unos índices de fiabilidad aceptables según los valores alcanzados en el alpha de Cronbach: .81 para la frecuencia, para los motivos y para las vías de consulta, y .82 para los contenidos.

- Difusión de noticias. Con el fin de registrar si los jóvenes se convierten, a su vez, en fuentes de la información que reciben. Al respecto se les pregunta por la frecuencia con que lo hacen ( $\alpha$ de Cronbach $=.79)$ y los medios y formas que utilizan para ello $(\alpha$ de Cronbach $=.73)$.

- Contraste de la información. Se intenta averiguar si los jóvenes se conforman con la primera información que les llega o si, por el contrario, recurren a medios y formas alternativas para contrastarla. En este sentido se analiza cuándo lo hacen y por qué lo hacen $(\alpha$ de Cronbach $=.84)$ y las vías preferidas para este cometido $(\alpha$ de Cronbach $=.76)$.

- Percepción sobre el grado de imparcialidad y de credibilidad. Entendido el primer concepto como sinónimo de neutralidad, es decir, la ausencia en las informaciones difundidas por los medios de tendencias a favor o en contra de determinadas líneas ideológicas en sus más diversos ámbitos. La credibilidad se corresponde con una cualidad subjetiva sobre la confianza que les inspira los distintos medios y formas de transmitir noticias en función de la veracidad o de otros parámetros (autoría, por ejemplo) que observan en sus contenidos. En cada uno de los campos se sumaron los ítems obteniendo un adecuado nivel de fiabilidad: coeficiente de .81 en la imparcialidad y .80 en el grado de credibilidad.

\section{Resultados}

\subsection{FRECUENCIA Y MOTIVOS DE CONSULTA}

De los escasos universitarios que reconocen no consultar nunca las noticias $(1,8 \%$ del total) el mayor porcentaje $(60 \%)$ se corresponde con el Centro político; el resto se sitúa a partes iguales en los dos posicionamientos moderados: Centro-Izquierda y Centro-Derecha. Del 98,2\% que sí se informan, el porcentaje mayor se registra en la frecuencia de una vez al día $(38,1 \%)$, seguido por este orden, de aquellos que no lo hacen siempre $(24,2 \%)$ y en dos ocasiones diarias $(21,7 \%)$ Tabla II. 
Tabla II. Contingencia Posición ideológica/Frecuencia diaria de consulta de noticias

\begin{tabular}{|c|c|c|c|c|c|c|c|}
\hline & \multicolumn{5}{|c|}{ Frecuencia de consulta } & \multirow[b]{2}{*}{ Total } \\
\hline & & Nunca & $\begin{array}{l}\text { No las miro } \\
\text { todos los días }\end{array}$ & $\begin{array}{l}\text { Una vez } \\
\text { al día }\end{array}$ & $\begin{array}{l}\text { Dos veces } \\
\text { al día }\end{array}$ & $\begin{array}{l}\text { Tres o más } \\
\text { veces al día }\end{array}$ & \\
\hline \multirow{3}{*}{$\begin{array}{l}\text { Extrema } \\
\text { Izquierda }\end{array}$} & \% Ideología &, $0 \%$ &, $0 \%$ & $100,0 \%$ &, $0 \%$ &, $0 \%$ & $100,0 \%$ \\
\hline & $\%$ F. consulta &, $0 \%$ &, $0 \%$ &, $9 \%$ &, $0 \%$ &, $0 \%$ &, $4 \%$ \\
\hline & $\%$ del total &, $0 \%$ &, $0 \%$ &, $4 \%$ &, $0 \%$ &, $0 \%$ &, $4 \%$ \\
\hline \multirow[t]{3}{*}{ Izquierda } & \% Ideología &, $0 \%$ & $23,1 \%$ & $28,2 \%$ & $25,6 \%$ & $23,1 \%$ & $100,0 \%$ \\
\hline & $\%$ F. consulta &, $0 \%$ & $13,2 \%$ & $10,3 \%$ & $16,4 \%$ & $22,5 \%$ & $13,9 \%$ \\
\hline & $\%$ del total &, $0 \%$ & $3,2 \%$ & $3,9 \%$ & $3,6 \%$ & $3,2 \%$ & $13,9 \%$ \\
\hline \multirow{3}{*}{$\begin{array}{l}\text { Centro- } \\
\text { Izquierda }\end{array}$} & \% Ideología & $1,0 \%$ & $26,5 \%$ & $38,2 \%$ & $18,6 \%$ & $15,7 \%$ & $100,0 \%$ \\
\hline & $\%$ F. consulta & $20,0 \%$ & $39,7 \%$ & $36,4 \%$ & $31,1 \%$ & $40,0 \%$ & $36,3 \%$ \\
\hline & $\%$ del total & $4 \%$ & $9,6 \%$ & $13,9 \%$ & $6,8 \%$ & $5,7 \%$ & $36,3 \%$ \\
\hline \multirow[t]{3}{*}{ Centro } & \% Ideología & $3,2 \%$ & $26,6 \%$ & $37,2 \%$ & $24,5 \%$ & $8,5 \%$ & $100,0 \%$ \\
\hline & $\%$ F. consulta & $60,0 \%$ & $36,8 \%$ & $32,7 \%$ & $37,7 \%$ & $20,0 \%$ & $33,5 \%$ \\
\hline & $\%$ del total & $1,1 \%$ & $8,9 \%$ & $12,5 \%$ & $8,2 \%$ & $2,8 \%$ & $33,5 \%$ \\
\hline \multirow{3}{*}{$\begin{array}{l}\text { Centro- } \\
\text { Derecha }\end{array}$} & \% Ideología & $2,9 \%$ & $17,1 \%$ & $42,9 \%$ & $20,0 \%$ & $17,1 \%$ & $100,0 \%$ \\
\hline & $\% \mathrm{~F}$. consulta & $20,0 \%$ & $8,8 \%$ & $14,0 \%$ & $11,5 \%$ & $15,0 \%$ & $12,5 \%$ \\
\hline & $\%$ del total &, $4 \%$ & $2,1 \%$ & $5,3 \%$ & $2,5 \%$ & $2,1 \%$ & $12,5 \%$ \\
\hline \multirow[t]{3}{*}{ Derecha } & \% Ideología & $0 \%$ & $10,0 \%$ & $60,0 \%$ & $20,0 \%$ & $10,0 \%$ & $100,0 \%$ \\
\hline & $\%$ F. consulta &, $0 \%$ & $1,5 \%$ & $5,6 \%$ & $3,3 \%$ & $2,5 \%$ & $3,6 \%$ \\
\hline & $\%$ del total &, $0 \%$ & $4 \%$ & $2,1 \%$ &, $7 \%$ &, $4 \%$ & $3,6 \%$ \\
\hline \multirow[t]{3}{*}{ Total } & \% Ideología & $1,8 \%$ & $24,2 \%$ & $38,1 \%$ & $21,7 \%$ & $14,2 \%$ & $100,0 \%$ \\
\hline & $\begin{array}{l}\% \text { dentro de } \\
\text { Frecuencia consulta }\end{array}$ & $100,0 \%$ & $100,0 \%$ & $100,0 \%$ & $100,0 \%$ & $100,0 \%$ & $100,0 \%$ \\
\hline & $\%$ del total & $1,8 \%$ & $24,2 \%$ & $38,1 \%$ & $21,7 \%$ & $14,2 \%$ & $100,0 \%$ \\
\hline
\end{tabular}

En líneas generales, los universitarios que prestan atención a la información responden mayoritariamente a la tendencia de Centro y de Izquierda. Al respecto es destacable el $40 \%$ que se posiciona en el Centro-Izquierda que consulta la información tres o más veces diarias, y el 37,7\%, de Centro, que lo hace dos o más veces diarias. En las tendencias conservadoras, el grupo más alto se sitúa en el sector moderado con un $15 \%$ que se informa tres o más veces diarias.

Los argumentos principales que esgrimen los encuestados para consultar las noticias son, por este orden, la necesidad de conocerlas de cara a su futuro profesional y estar al tanto de la actualidad. Como tercera causa, únicamente los que se consideran de Izquierda entienden que este hábito es fundamental para la toma de decisiones políticas o de otra índole; los que se perciben dentro de la CentroDerecha lo igualan al conocimiento de un ámbito concreto, mientras que el resto prioriza esta última causa frente a la toma de decisiones (Gráfico 1). 
Grafico 1. Motivos que les induce a consultar noticias en función de la tendencia ideológica

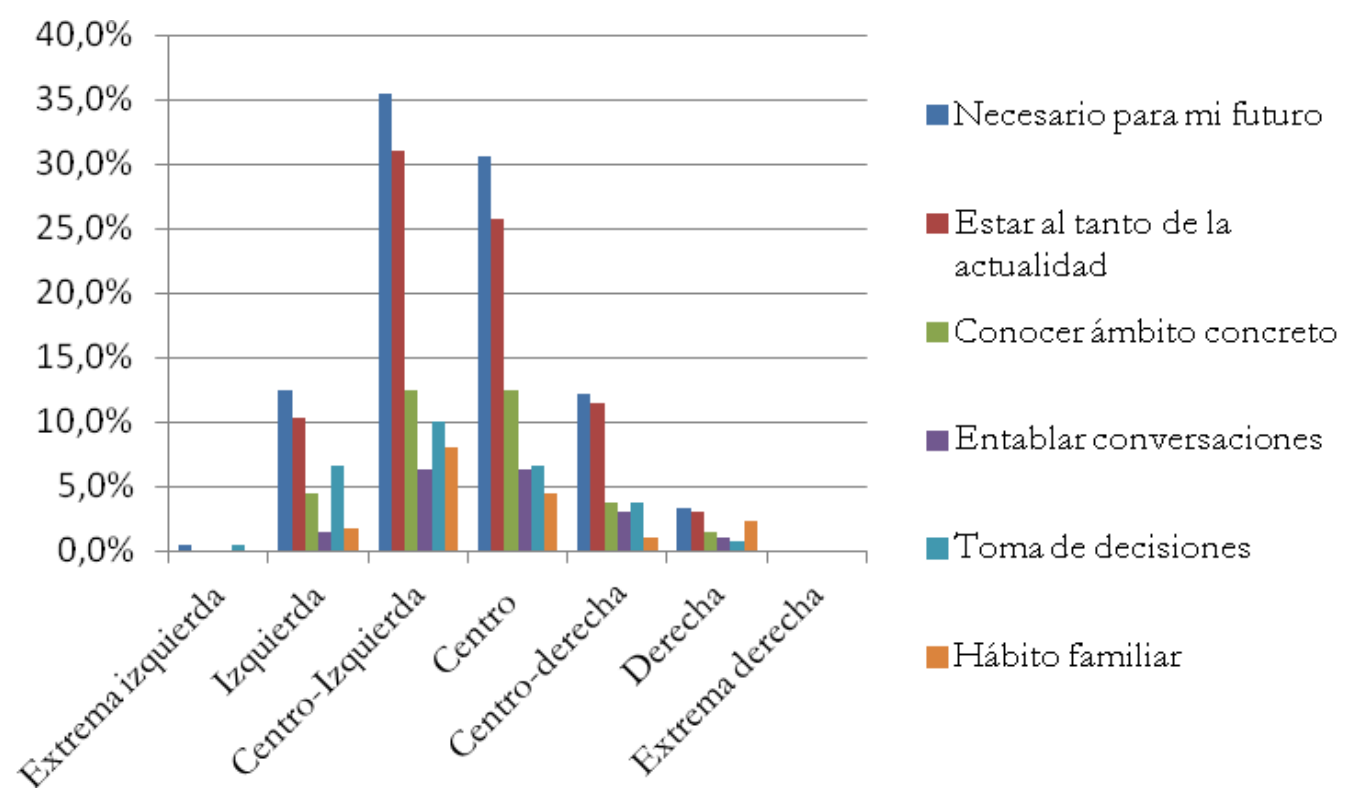

Fuente: elaboración propia.

\subsection{CONTENIDOS PREFERIDOS}

Los ámbitos relacionados con Sociedad $(25,8 \%)$ y, en segundo lugar, los vinculados a la Política $(18,5 \%)$ son los elegidos mayoritariamente en todos los grupos ideológicos. Los valores máximos se registran en los jóvenes situados en el Centro-Izquierda y Centro que optan por las informaciones sociales con unos respectivos $8,3 \%$ y $9,7 \%$ sobre el total.

En el polo opuesto y sin contar con las informaciones de ámbito indeterminado (Otros), las noticias del corazón se sitúan en la última opción de las preferencias de gran parte de los grupos ideológicos. Solo los encuestados que se inclinan por el Centro-Derecha las colocan por encima de Medio Ambiente, y los que optan por la Derecha prefieren la prensa rosa a las informaciones de CienciaTecnología y medioambientales. Al margen de estas primeras opciones, se registran un equilibrio superior en las preferencias recogidas en los jóvenes que se declaran con una tendencia algo menos centrista. $\mathrm{Al}$ respecto, los universitarios que se auto-posicionan en la Izquierda optan por igual $(11,8 \%)$ entre las secciones de Economía, Deportes, Ciencia-Tecnología y Medio Ambiente; y aquellos encuestados que se inclinan por partidos de Derecha registran el mismo porcentaje $(7,1 \%)$ en relación con Economía, Ciencia-Tecnología y Medio Ambiente. En este sentido, lo único que diferencia a estos dos grupos es que los conservadores se inclinan sensiblemente más por los Deportes $(10,8 \%)$.

Los jóvenes de centro y los más cercanos a esta posición muestran una mayor diversidad entre las noticias que consultan: en ningún caso se observa una igualdad porcentual entre una temática y otra. En cualquiera de los casos no hay una inclinación temática correlacionada con la posición ideológica. 


\subsection{MEdios de CONSUlta}

La televisión convencional marca unos datos muy significativos al alza como medio para la consulta de noticias, sobre todo entre los de Centro-Izquierda (82,6\%) y Centro (90,2\%). En el conjunto de los medios tradicionales, le sigue a larga distancia la prensa escrita, aunque en este caso son los que se ubican en el Centro-Derecha $(16,1 \%)$ y en la Derecha $(11,1 \%)$ quienes prefieren esta opción. La radio tiene una presencia casi nula, incluso presenta registros más bajos entre los que no se informan a través de estos medios. El desinterés por la información radiofónica se refuerza entre los jóvenes de Centro-Derecha y de Derecha.

A excepción de un único universitario que apuesta por el Centro político, el resto de encuestados utiliza alguna vía online para informarse de la actualidad, principalmente a través de las redes sociales $(35,3 \%)$ y la prensa online $(29,9 \%)$. Este orden de preferencia se da en todas las tendencias ideológicas menos en la Izquierda que opta en una media sensiblemente mayor por la prensa online: $38,8 \%$ frente a $36,3 \%$ alcanzado por las redes sociales. El único caso registrado de Extrema Izquierda también sigue esta tendencia y afirma que solo consulta las noticias de forma digital a través de la prensa y de aplicaciones de mensajería como WhatsApp (Tabla III).

Resulta paradójico que, salvando los porcentajes detectados en los periódicos online, otros medios digitales destinados esencialmente a la difusión de noticias alcanzan unos valores inferiores a otras formas online cuyo destino es más variado. En este sentido, la radio online, igual que ocurría con la versión tradicional, vuelve a ser el medio menos consultado. Mientras que la TV online tiene una aceptación contraria a su forma convencional: el 4,1\% recurre a este medio, porcentaje idéntico al alcanzado por los blogs.

En las otras formas que no están consideradas únicamente para informarse de la actualidad, los resultados son notablemente superiores: además de las citadas redes sociales, las aplicaciones de mensajería instantánea registran un $12,9 \%$ de recurrencia y los buscadores un 10,9\%. Esta preferencia se da en todos los grupos ideológicos con especial incidencia en el centro y en sus tendencias más próximas (Centro-Izquierda, Centro-Derecha). 
Tabla III. Contingencia posición ideológica/forma digital

\begin{tabular}{|c|c|c|c|c|c|c|c|c|c|c|c|}
\hline & \multicolumn{9}{|c|}{ Forma digital } & \multirow[b]{2}{*}{ Total } \\
\hline & & Ninguna & $\begin{array}{l}\text { Radio } \\
\text { online }\end{array}$ & $\begin{array}{c}\text { TV } \\
\text { online }\end{array}$ & $\begin{array}{l}\text { Prensa } \\
\text { online }\end{array}$ & Blogs & \begin{tabular}{|l|} 
Redes \\
Sociales
\end{tabular} & $\begin{array}{c}\text { Servicios } \\
\text { Mensajería }\end{array}$ & Buscadores & Otros & \\
\hline \multirow[t]{3}{*}{$\begin{array}{l}\text { Extrema } \\
\text { Izquierda }\end{array}$} & $\begin{array}{l}\text { \% Ideolo- } \\
\text { gía }\end{array}$ &, $0 \%$ &, $0 \%$ &, $0 \%$ & $50,0 \%$ &, $0 \%$ & $50,0 \%$ &, $0 \%$ &, $0 \%$ &, $0 \%$ & $100,0 \%$ \\
\hline & $\begin{array}{l}\% \mathrm{~F} . \\
\text { digital }\end{array}$ &, $0 \%$ &, $0 \%$ &, $0 \%$ &, $5 \%$ &, $0 \%$ &, $4 \%$ &, $0 \%$ &, $0 \%$ &, $0 \%$ &, $3 \%$ \\
\hline & $\begin{array}{l}\% \text { del } \\
\text { total }\end{array}$ &, $0 \%$ &, $0 \%$ &, $0 \%$ &, $1 \%$ &, $0 \%$ &, $1 \%$ &, $0 \%$ &, $0 \%$ &, $0 \%$ &, $2 \%$ \\
\hline \multirow[t]{3}{*}{ Izquierda } & $\begin{array}{l}\text { \% Ideolo- } \\
\text { gía }\end{array}$ &, $0 \%$ & $2,5 \%$ & $3,7 \%$ & $38,8 \%$ & $5,0 \%$ & $36,3 \%$ & $8,7 \%$ & $5,0 \%$ & & $100,0 \%$ \\
\hline & $\begin{array}{l}\% \mathrm{~F} . \\
\text { digital }\end{array}$ &, $0 \%$ & $20,0 \%$ & $11,5 \%$ & $16,4 \%$ & $15,4 \%$ & $12,9 \%$ & $8,5 \%$ & $5,8 \%$ &, $0 \%$ & $12,6 \%$ \\
\hline & $\begin{array}{l}\% \text { del } \\
\text { total }\end{array}$ &, $0 \%$ &, $3 \%$ &, $5 \%$ & $4,9 \%$ &, $6 \%$ & $4,6 \%$ & $1,1 \%$ &, $6 \%$ &, $0 \%$ & $12,6 \%$ \\
\hline \multirow[t]{3}{*}{$\begin{array}{l}\text { Centro- } \\
\text { Izquierda }\end{array}$} & $\begin{array}{l}\text { \% Ideolo- } \\
\text { gía }\end{array}$ &, $0 \%$ & $2,8 \%$ & $3,2 \%$ & $27,2 \%$ & $3,7 \%$ & $35,9 \%$ & $12,4 \%$ & $13,9 \%$ &, $9 \%$ & $100,0 \%$ \\
\hline & $\begin{array}{l}\% \mathrm{~F} . \\
\text { digital }\end{array}$ &, $0 \%$ & $60,0 \%$ & $26,9 \%$ & $31,2 \%$ & $30,8 \%$ & $34,7 \%$ & $32,9 \%$ & $43,5 \%$ & $40,0 \%$ & $34,3 \%$ \\
\hline & $\begin{array}{l}\% \text { del } \\
\text { total }\end{array}$ &, $0 \%$ &, $9 \%$ & $1,1 \%$ & $9,3 \%$ & $1,3 \%$ & $12,4 \%$ & $4,3 \%$ & $4,7 \%$ &, $3 \%$ & $34,3 \%$ \\
\hline \multirow[t]{3}{*}{ Centro } & $\begin{array}{l}\text { \% Ideolo- } \\
\text { gía }\end{array}$ &, $5 \%$ & & $5,1 \%$ & & $5,6 \%$ & & $13,4 \%$ & $9,7 \%$ & & $100,0 \%$ \\
\hline & $\begin{array}{l}\% \mathrm{~F} . \\
\text { digital }\end{array}$ & $100 \%$ & $20,0 \%$ & $42,3 \%$ & $32,8 \%$ & $46,2 \%$ & $33,8 \%$ & $35,4 \%$ & $30,4 \%$ & $40,0 \%$ & $34,1 \%$ \\
\hline & $\begin{array}{l}\% \text { del } \\
\text { total }\end{array}$ &, $2 \%$ &, $3 \%$ & $1,7 \%$ & $9,8 \%$ & $1,9 \%$ & $12,0 \%$ & $4,6 \%$ & $3,3 \%$ &, $3 \%$ & $34,1 \%$ \\
\hline \multirow[t]{3}{*}{$\begin{array}{l}\text { Centro- } \\
\text { Derecha }\end{array}$} & $\begin{array}{l}\text { \% Ideolo- } \\
\text { gía }\end{array}$ &, $0 \%$ &, $0 \%$ & $4,2 \%$ & $30,5 \%$ & $2,1 \%$ & $32,6 \%$ & $16,8 \%$ & $12,6 \%$ & $1,1 \%$ & $100,0 \%$ \\
\hline & $\begin{array}{l}\% \mathrm{~F} . \\
\text { digital }\end{array}$ &, $0 \%$ &, $0 \%$ & $15,4 \%$ & $15,4 \%$ & $7,6 \%$ & $13,8 \%$ & $19,5 \%$ & $17,4 \%$ & $20,0 \%$ & $15 \%$ \\
\hline & $\begin{array}{l}\% \text { del } \\
\text { total }\end{array}$ &, $0 \%$ &, $0 \%$ &, $6 \%$ & $4,6 \%$ &, $3 \%$ & $4,9 \%$ & $2,5 \%$ & $1,9 \%$ & $2 \%$ & $15 \%$ \\
\hline \multirow[t]{3}{*}{ Derecha } & $\begin{array}{l}\text { \% Ideolo- } \\
\text { gía }\end{array}$ &, $0 \%$ &, $0 \%$ & $4,3 \%$ & $30,4 \%$ &, $0 \%$ & $43,5 \%$ & $13,0 \%$ & $8,7 \%$ &, $0 \%$ & $100,0 \%$ \\
\hline & $\begin{array}{l}\% \\
\text { F.digital }\end{array}$ &, $0 \%$ &, $0 \%$ & $3,9 \%$ & $3,7 \%$ &, $0 \%$ & $4,4 \%$ & $3,7 \%$ & $2,9 \%$ &, $0 \%$ & $3,6 \%$ \\
\hline & $\begin{array}{l}\% \text { del } \\
\text { total }\end{array}$ &, $0 \%$ &, $0 \%$ &, $2 \%$ & $1,1 \%$ &, $0 \%$ & $1,6 \%$ &, $5 \%$ &, $3 \%$ &, $0 \%$ & $3,6 \%$ \\
\hline \multirow[t]{3}{*}{ Total } & $\begin{array}{l}\text { \% Ideolo- } \\
\text { gía }\end{array}$ & & & & & $4,1 \%$ & $35,3 \%$ & $12,9 \%$ & $10,9 \%$ &, $8 \%$ & \\
\hline & $\begin{array}{l}\% \text { Forma } \\
\text { digital }\end{array}$ & $100 \%$ & $100 \%$ & $100 \%$ & $100 \%$ & $100,0 \%$ & $100,0 \%$ & $100,0 \%$ & $100 \%$ & $100,0 \%$ & $100,0 \%$ \\
\hline & $\begin{array}{l}\% \text { del } \\
\text { total }\end{array}$ & , 2\% & $1,6 \%$ & $4,1 \%$ & $29,9 \%$ & $4,1 \%$ & $35,3 \%$ & $12,9 \%$ & $10,9 \%$ &, $8 \%$ & $100,0 \%$ \\
\hline
\end{tabular}




\subsection{FRECUENCIA Y FORMAS PARA DIFUNDIR NOTICIAS}

Excepto los jóvenes auto-posicionados en la Derecha política, que sobre todo lo hacen ocasionalmente, la mayoría asegura que casi siempre difunden a su entorno las noticias que reciben. Solo uno de Centro-Izquierda reconoce que no lo hace nunca y 5 de esta misma orientación ( $62,5 \%$ sobre esta frecuencia) dicen que casi nunca se erigen como fuentes de la información entre su círculo; el resto que se localiza en esta periodicidad se reparte entre Centro-Izquierda (25\%) y Centro (12,5\%).

En cuanto a las formas que utilizan para difundir las noticias en su entorno (al margen del posicionado en Extrema-Izquierda que asegura utilizar únicamente las redes sociales), prácticamente todos los grupos mantienen de forma prioritaria el cara a cara para llevar a cabo este cometido, con una oscilación entre el 35,6\% de los centristas y un 40,2\% de los que se engloban en la Izquierda (Gráfico 2). El único grupo que se desmarca es el de la Derecha que, con un significativo porcentaje de $47,3 \%$, prefiere las redes sociales; mientras que la difusión directa en el entorno físico es utilizada por el 26,3\% de los jóvenes conservadores.

Los servicios de mensajería como Whats App son utilizados como segunda opción por los universitarios de Centro (30,2\%) y de Centro-Derecha (28,9\%); mientras que los de Izquierda (23,2\%), Centro-Izquierda $(26,6 \%)$ y Derecha $(21,1 \%)$ sitúan esta vía en la tercera posición, por debajo de las redes sociales. El teléfono y, sobre todo, los blogs son apenas utilizados por todos los grupos. Al respecto, los dos grupos situados en el ala izquierda despuntan sensiblemente en el empleo de la vía telefónica, aunque con valores que no alcanzan en ninguno de los casos los 10 puntos porcentuales: 8,9\% para los de Centro-Izquierda y 7,3\% entre los de Izquierda. Para el resto de los grupos apenas llega a un $5 \%$.

El blog tampoco se convierte en una opción recurrente por los universitarios. De hecho, ninguno posicionado en la Derecha afirma utilizarlo y, en el resto de las tendencias, la horquilla se sitúa entre el 0,5\% de los estudiantes de Centro-Izquierda y el 1,3\% de los que se sitúan en Centro-Derecha.

Gráfico 2. Formas de difusión de noticias en función de la tendencia*

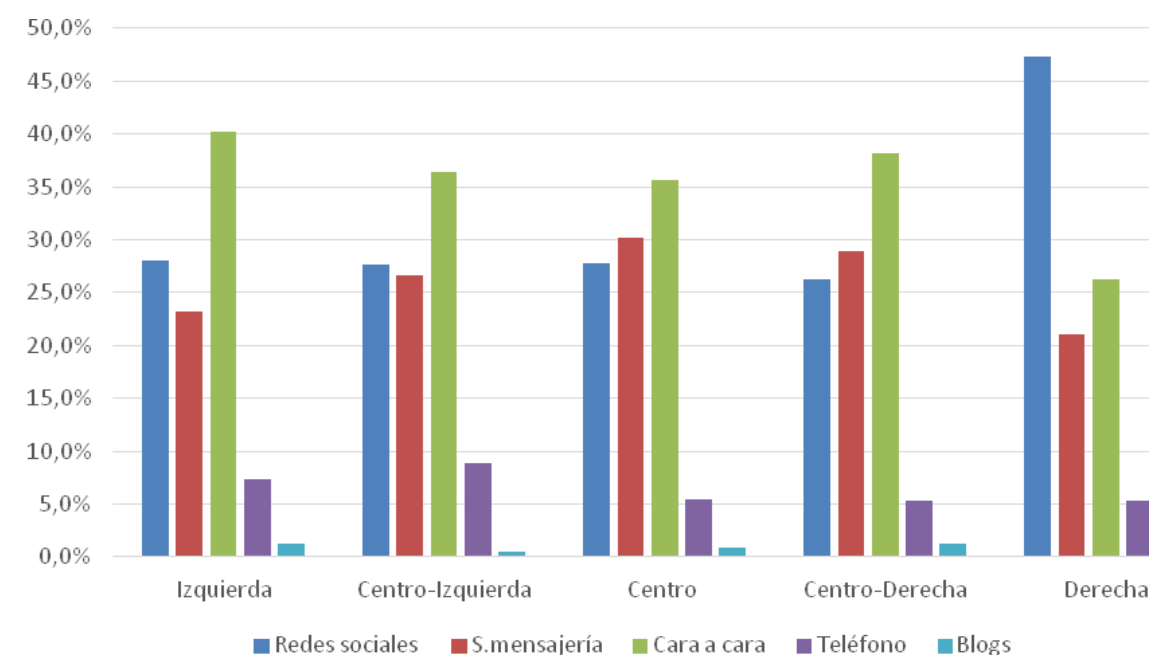

Fuente: elaboración propia. Para la construcción de este gráfico se ha suprimido la extrema-izquierda dado que la variable para el único posicionado en esta tendencia refleja un $100 \%$ en redes sociales, porcentaje que desvirtúa una cómoda visualización del resto de ítems. 


\subsection{CONTRASTE DE NOTICIAS. MOTIVOS Y FORMAS EMPLEADAS}

En líneas generales, los encuestados manifiestan contrastar las noticias; un 47\% lo hace siempre; y $42,6 \%$ mantiene este hábito ocasionalmente. El 10,4\% restante que reconoce no hacerlo pertenece fundamentalmente a los tres grupos situados en el Centro, motivados en este orden, por su confianza en el primer medio que les informa y por la falta de tiempo para consultar otras fuentes de consulta.

Las causas que incitan al $47 \%$ de los jóvenes que siempre contrasta las noticias son diversas en función de la tendencia ideológica. No se ha observado un patrón de comportamiento definido: los que prefieren el Centro (18,8\%) y Derecha política $(2,4 \%)$ aseguran que la razón principal para consultar otras vías es completar más datos en relación con la información. Mientras que el resto de los grupos, especialmente los dos grupos de Izquierda, tienen como intención confirmar las versiones que les ofrece el primer medio: $19,5 \%$ para los moderados de esta tendencia y $8,6 \%$ para los agrupados en la Izquierda.

Entre el 42,6\% de los universitarios que lo hacen ocasionalmente sí que se observa una correspondencia con la orientación: los de Centro $(17,8 \%)$ y los situados en ambas franjas de la Derecha (6,9\% los moderados y 3,5\% los más conservadores) están motivados para contrastar las noticias cuando la información incide en su interés personal, mientras que en los dos grupos situados a la Izquierda optan por consultar distintas versiones de la información si su contenido entraña cierta polémica. Esta diferenciación entre ideologías se refuerza al observar que ninguno de los universitarios inclinados a la Derecha política contrasta la información que suponga algún tipo de controversia.

La prensa online y, en segundo lugar, la televisión convencional son las formas más utilizadas por los jóvenes situados en todas las tendencias de Izquierda; preferencias que también se observan en los de Centro y en los posicionados a la Derecha. Quienes se encuentran en el Centro-Derecha, aunque también escogen prioritariamente ambos medios para contrastar las noticias, optan más por la televisión tradicional (4,1\% del total). Un porcentaje sensiblemente superior al registrado en la prensa digital $(3,3 \%)$. En esta vía de contraste se sitúa el mayor porcentaje alcanzado de todos los medios $(27,3 \%)$, con una especial incidencia en los de Centro-Izquierda, que alcanzan el baremo más alto con un 9,7\% del total.

La radio, en ninguna de sus dos versiones, es prácticamente utilizada para este fin por ninguno de los grupos. Este escaso apego toma especial fuerza con el formato digital entre los jóvenes englobados en los grupos más radicales de la Izquierda y los de Centro-Derecha: ninguno de ellos utiliza este medio para contrastar y solo 4 del resto de los grupos sí que recurren a ella. Tampoco tienen gran relevancia la TV online, al contrario de lo que ocurre con su versión tradicional; solo recurren a este medio digital un $0,8 \%$ del total, repartidos de forma muy similar entre todos los grupos, a excepción de los situados en la derecha y en extrema-izquierda que registran valores nulos.

Los blogs, igualmente, no son seguidos prioritariamente por ninguno de los grupos. Sí resulta significativo que las aplicaciones de mensajería instantánea, cuya función primordial es establecer comunicaciones con el entorno, mucho más allá que la difusión de la actualidad, se sitúan en la cuarta posición en las preferencias de los situados en Centro-Derecha y en la quinta en el resto de los grupos. Las redes sociales también adquieren una notable significación y se sitúan en la tercera posición por parte de todos los grupos ideológicos considerados, a excepción del único que responde a la ExtremaIzquierda, quien solo utiliza la prensa online para este cometido. 


\subsection{PERCEPCIÓN SOBRE LA IMPARCIALIDAD Y LA CREDIBILIDAD}

Aparte del universitario de Extrema-Izquierda, que no contesta a su percepción sobre los medios tradicionales y que la máxima valoración que otorga es la de bastante imparcial a la prensa online (bajo una escala de Likert en la que 1 es considerado nada imparcial y 5 totalmente imparcial), entre todos los medios y formas que los universitarios tienen para recuperar información de actualidad, solo dos de ellos guardan una correlación entre la percepción de imparcialidad y la tendencia ideológica. Ambos casos corresponden a versiones tradicionales: la prensa y, sobre todo, la televisión, que son consideradas más imparciales cuanto más radical sea la posición ideológica hacia la Derecha, de tal forma que el medio televisivo es valorado en una horquilla que parte de una media de 2,1 calificado por los jóvenes de Izquierda y un 3 por los de Derecha; y la franja por la que discurre la neutralidad de los periódicos impresos oscila entre el 2 señalado por los que se inclinan por la Izquierda política y el 2,5 registrado en el Centro-Derecha y en la Derecha. Como contraste, en la versión convencional de la radio se observan diversas fluctuaciones que no guardan correlación con el auto-posicionamiento político (Gráfico 3).

Gráfico 3. Percepción de la imparcialidad en medios convencionales

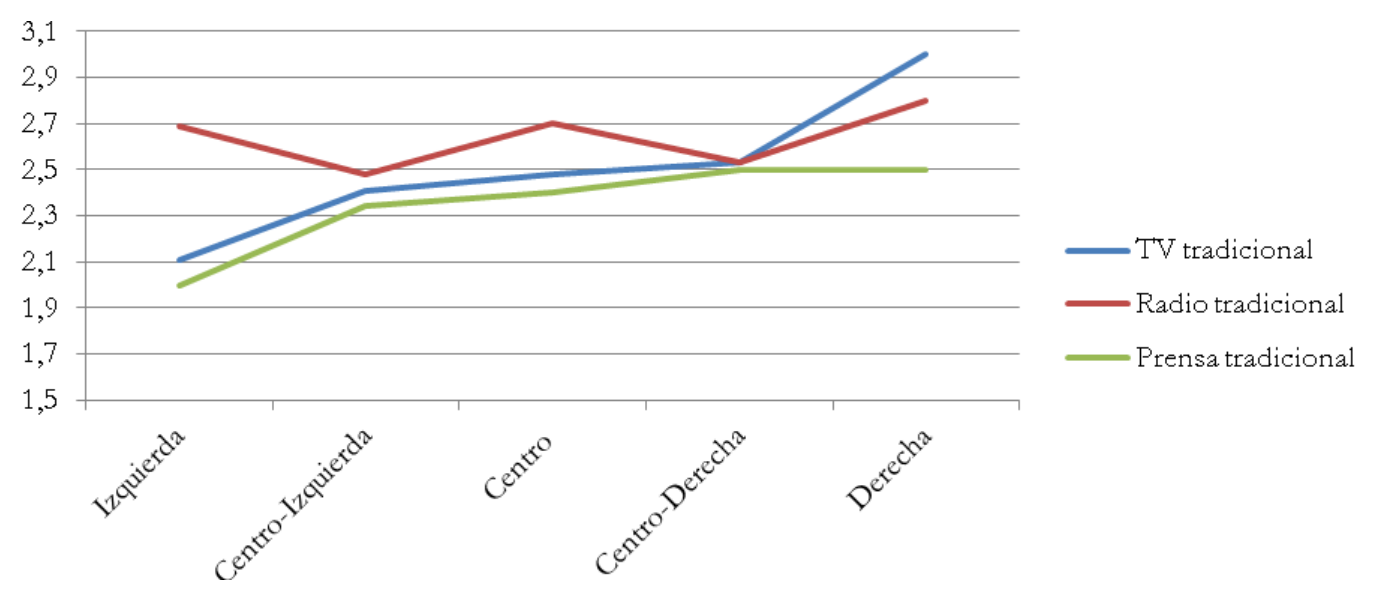

Fuente: elaboración propia.

Para las distintas vías incluidas en Internet que los jóvenes usan para consultar información, no se observa una correlación con la ideología: mientras que los situados en la Izquierda y los de Centro consideran la prensa online como el modo de informarse más imparcial, los dos grupos más moderados de ambas tendencias y los de derecha creen que la mayor neutralidad se halla en las redes sociales (Gráfico 4). 
Gráfico 4. Percepción de la imparcialidad en medios online y otras formas de consulta por Internet

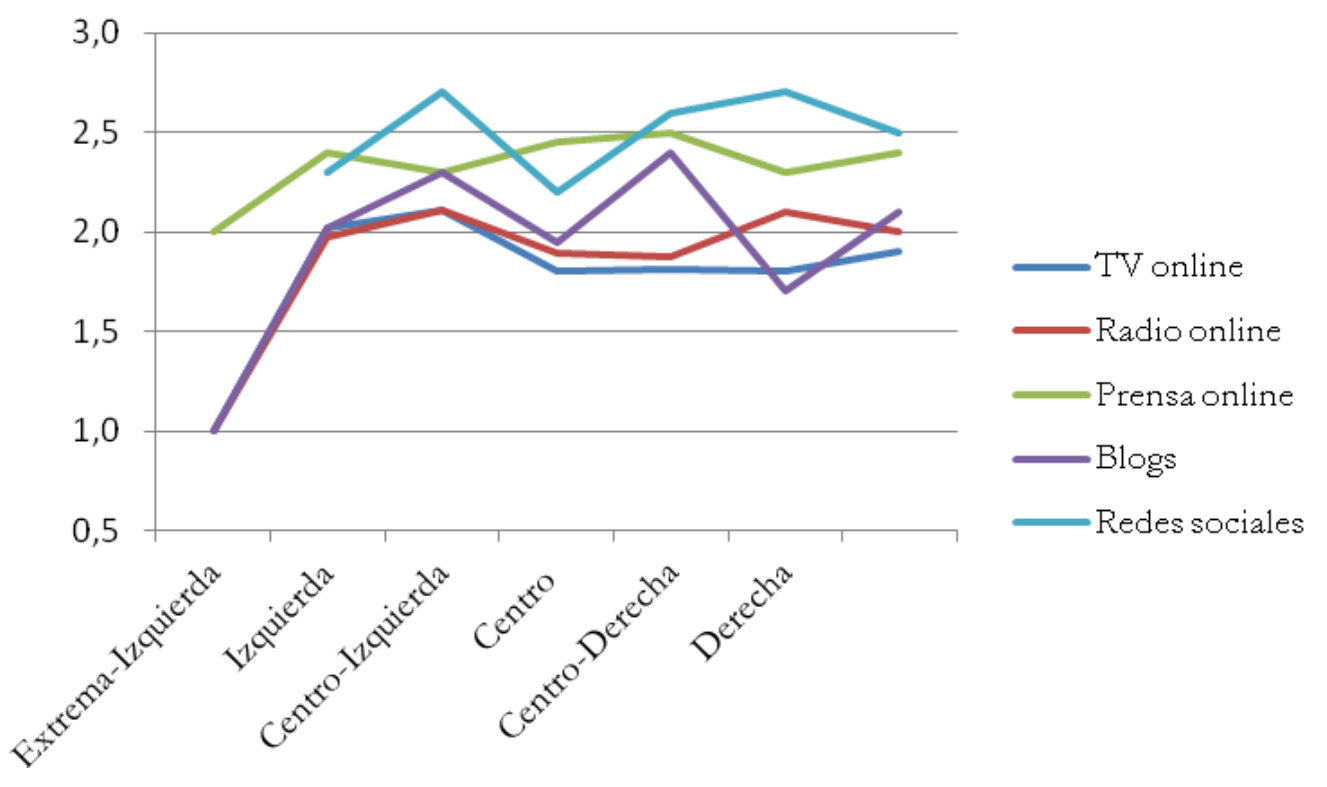

Fuente: elaboración propia.

En cualquiera de los casos, tanto en las versiones offline como online, los valores son moderados tendiendo a la baja; la menor puntuación $(1,7)$ proviene de los jóvenes de Derecha hacia los blogs; mientras que la calificación más alta, un 3 de media, es otorgada también por el grupo de esta misma tendencia a la televisión convencional.

Examinada la credibilidad con los mismos valores que la imparcialidad (1 nada creíble, 7 totalmente creíble) los jóvenes otorgan, en general, unas puntuaciones mayores que a la neutralidad que perciben en las vías que emplean para la consulta de noticias. De hecho, en varios de los casos y dependiendo de las tendencias ideológicas, la confianza supera los 3 puntos de media, fundamentalmente en dos de los medios tradicionales: la radio offline y la prensa escrita. Ambos son vistos con esta confianza por todos los grupos ideológicos salvo por la Izquierda, quienes, en contraste, son los únicos que ofrecen una media algo mayor de 3 a las redes sociales. La prensa online también obtiene estas puntuaciones notables entre la mayor parte de los universitarios excepto los posicionados en el Centro-Izquierda que conceden una media de credibilidad a este medio de un 2,8.

Las vías para informarse peor calificadas son la televisión y la radio online, y sobre todo los blogs; en este último caso se observa el único valor por debajo del 2 de todos los grupos ideológicos establecidos: los jóvenes de derechas les conceden solo un 1,7 de confianza. Por el contrario, la mayor puntuación de credibilidad $(3,6)$ es otorgada a la prensa tradicional por los jóvenes que se sitúan en Centro-Derecha y le sigue muy de cerca $(3,5)$ la televisión convencional que también mantiene este alto grado de confianza entre los posicionados en la misma tendencia.

Finalmente se observa una única correlación entre la tendencia y el grado de credibilidad que corresponden a la radio online: la línea medial avanza hacia la Derecha desde el 2,1 que conceden los izquierdistas hasta el 2,6 de los más conservadores. Para el resto de fuentes de consulta, los universitarios tienen posiciones más diversas. 


\section{CONCLUSIONES Y DISCUSIÓN}

Este trabajo tiene como objetivo genérico aproximarse a las interacciones y relaciones que se establecen entre el posicionamiento político de los jóvenes españoles y el comportamiento mediático digital.

La primera de las cuestiones abordadas en la investigación está relacionada con la frecuencia, los motivos, así como los medios y modos que emplean los jóvenes para el acceso y chequeo de la información de actualidad. En este sentido, sí se observa una correlación entre el posicionamiento político o ideológico, en este caso los que declaran preferir el Centro-Izquierda, y la mayor atención a las noticias en Internet. Sin embargo, no se encuentra una relación directa del posicionamiento político con las razones para la consulta de noticias.

En cuanto a los contenidos consultados, aunque se observan diferencias de comportamiento no se pueden extraer conclusiones sobre la existencia de patrones diferenciados de comportamiento a partir de la opción política. En términos generales, son los ámbitos de Sociedad (25,8\%) y Política $(18,5 \%)$ los de mayor impacto entre los jóvenes encuestados. Al mismo tiempo, sigue siendo la televisión convencional el medio al que más se acude para informarse, sobre todo en el caso de los encuestados que se reconocen como de Centro-Izquierda o Centro. A continuación aparece la prensa escrita.

En términos generales, el acceso en línea a las noticias se realiza a través de las redes sociales $(35,3 \%)$ o bien mediante la prensa digital $(29,9 \%)$. No obstante, este orden se revierte si hacemos referencia a los que optan por un posicionamiento de izquierdas, puesto que muestran una preferencia mayor por la prensa digital (38,8 frente al 36,3\% de las redes sociales).

En segundo lugar, este trabajo se ha detenido en cómo los jóvenes difunden las noticias a las que tienen acceso y su relación con sus convicciones políticas. Así, se puede ver que un amplio porcentaje de los encuestados ayudan a la propagación de las noticias que les resultan de interés, una pauta en la línea de lo concluido por Jiménez y Gaitán Caballero (2013). En lo que se refiere a los medios o procedimientos que emplean a tal efecto, se observa una diferencia evidente: la mayor parte de los encuestados prefieren el cara a cara. La única diferencia viene de la mano de quienes se muestran más cercanos a las opciones conversadoras que mencionan las redes sociales como primera opción (47,3\%), frente al cara a cara, con un 26,3\%. En términos generales, las redes sociales o las aplicaciones como el WhatsApp aparecen como segunda o tercera opción global, en función de la orientación ideológica.

Por otro lado, el tercer objetivo ha sido conocer la percepción que los jóvenes tienen de los diferentes canales para acceder a la información de actualidad, en términos de imparcialidad y grado de confianza (credibilidad). En lo que afecta a la imparcialidad, no se ha encontrado una correlación directa con el auto-posicionamiento político, aunque sí algunas divergencias. Así, los tendentes a la Izquierda y al Centro apuestan por la prensa digital como la más imparcial, mientras que los más conservadores afirman que las redes sociales tienen un mayor grado de neutralidad. A la hora de analizar la credibilidad, sobresalen la radio tradicional y la prensa escrita, salvo para las personas que demuestran una tendencia de Izquierda, que optan por las redes sociales. Se concluye que la credibilidad no está asociada al canal comunicativo por lo que otras variables, como las definidas por García Avilés et al. (2014) como el prestigio del medio o la firma pueden mantener su vigencia.

A pesar del carácter no representativo de la muestra seleccionada, este trabajo presenta datos relevantes que complementan otras investigaciones (Qayyum, et al., 2010; Casero-Ripollés, 2012; o Rayén Condeza, et al., 2014; entre otros) sobre el consumo mediático de los jóvenes, en este caso españoles. La lectura de este artículo se debe realizar teniendo en cuenta que se ha focalizado en jóvenes universitarios, lo que puede producir sesgos en los resultados y conclusiones al no considerar a todo el colectivo analizado. Junto a este aspecto, otro sobre el que vale la pena detenerse para la futura inves- 
tigación y la reflexión es la clara disociación encontrada entre el uso mediático y los criterios de credibilidad aplicados. Por otra parte, también se debe profundizar en las categorías políticas empleadas, así como en la incidencia de temas polémicos concretos representativos de la ideología de los sujetos estudiados.

Si bien se ha observado que hay un alto porcentaje de jóvenes que contrastan la primera fuente que consultan, sería interesante conocer también hasta qué punto indagan si los contenidos están legitimados y son acordes con los hechos producidos. Esta interrogante es relevante porque más de un tercio de los jóvenes encuestados recurren a las redes sociales para informarse de primera mano. Este hallazgo sugiere que les preocupa mínimamente la autoría de la información y, por tanto, la autenticidad de los contenidos derivado de la sobreabundancia de fuentes (no todas fiables) que se pueden encontrar en las redes. En este contexto es discutible si los jóvenes tienen una correcta alfabetización mediática también para llevar a cabo este cometido, la consulta de noticias.

Además, hay que tener en cuenta que, aunque la cifra de un $10,4 \%$ no es muy alta entre aquellos que nunca contrastan la información de actualidad, sí es significativo su escaso interés por saber si la información refleja la realidad correctamente. En paralelo, y como nuevo punto de discusión, sería importante ver si profundizan en los contenidos o simplemente se conforman con la lectura de titulares. Esta indagación se podría correlacionar igualmente con su autopercepción sobre el conocimiento que tienen de la actualidad.

Este artículo está vinculado al proyecto «Redes sociales, adolescentes y jóvenes: convergencia de medios y cultura digital» (CSO2016-74980-C2-2-R) financiado por el Ministerio de Economía, Industria y Competitividad de España (2017-2020).

\section{BIBLIOGRAFÍA}

Bernal, A. I. (2015). Tecnología, redes sociales y periodismo: ¿Pluralidad informativa o efecto bumerán? Cuadernos. Info, 36, 191-205. doi: https://doi.org/10.7764/cdi.36.647

Bobbio, N. (1995). Derecha e Izquierda. Razones y significados de una distinción politica. Madrid: Taurus Pensamiento.

Carlsson, U. (2011). Young People in the Digital Media Culture. En C. Von Feilitzen; U. Carlsson y C. Bucht (Eds.), New Questions, New Insights, New Approaches (pp. 15-18). Göteborg: The International Clearinghouse on Children, Youth and Media. Nordicom.

Casero-Ripollés, A. (2012). Más allá de los diarios: el consumo de noticias de los jóvenes en la era digital. Comunicar, 20(39), 151-158. doi: https://doi.org/10.3916/C39-2012-03-05

Casero-Ripollés, A., Ortells-Badenes, S. y Rosique-Cedillo, G. (2014). Consecuencias democráticas de la disolución de las fronteras entre información, entretenimiento y privacidad en la era digital. Telos, 99, 45-54.

Catalina-García, B., García Jiménez, A. y Montes Vozmediano, M. (2015). Jóvenes y consumo de noticias a través de Internet y los medios sociales/Youths and consumption of news via Internet and social media. Historia y Comunicación Social, 20(2), 601-619. doi: https://doi.org/10.5209/rev HICS.2015.v20.n2.51402

García Avilés, J.A.; Navarro Maillo, F. y Arias Robles, F. (2014). La credibilidad de los contenidos informativos en internet para los 'nativos digitales': estudio de caso. Palabra Clave, 17(3), 875-894. doi: https://doi.org/10.5294/pacla.2014.17.3.13 
Espinoza, P. y Calderón, A. (2006). Ideología política, valores culturales y miedo a la muerte: su impacto después de los atentados del 11 de marzo. Psicología Política, 32, 33-58. Recuperado de http://www.uv.es/garzon/psicologia\%20politica/N32-3.pdf

Gómez Rubio, L. y López Vidales, N. (2016). Información televisiva y movilidad. Análisis de las noticias más seguidas a través de Twitter. Fonseca, Journal of Communication, 13, 11-31. doi: $10.14201 /$ fjc2016131131

Gutiérrez-Coba, L., Gómez-Díaz, J. A., Salgado-Cardona, A., Estrada-Gutiérrez, J. y Ramírez Méndez, M. J. (2012) Criterios y hábitos de consumo de información periodística online en Colombia. El profesional de la información, 21(3), 261-267. doi: 10.3145/epi.2012.may.06

Hanusch, F. (2013). Sensationalizing death? Graphic disaster images in the tabloid and broadsheet press. European Journal of Communication, 28(5), 497-513. doi: https://doi.org/10.1177/0267323113491349

Jiménez, C. y Gaitán Caballero, B. N. (2013). Análisis de los factores que motivan a los estudiantes de la Universidad Autónoma de Occidente a difundir contenidos virales en la Red. Colombia, Santiago de Cali, Universidad Autónoma de Occidente. Recuperado de https://red.uao.edu.co/bitstream/10614/5127/1/TCP01511.pdf.

Kim, K.-S., Sin, S.-C. J. y Yoo-Lee, E. (2014). Undergraduates' use of social media as information sources. College \& Research Libraries, 75(4), 442-457.

López-Meri, A (2016). Periodismo en Twitter. La contribución de los usuarios al flujo informativo. Cuadernos.Info, 39, 241-257. doi: https://doi.org/10.7764/cdi.39.825

Mellado Ruiz, C., López-Rabadán, P. y Elortegui, C. (2016) ¿(Para) ciudadanos o espectadores? Periodismo político chileno y sus modelos profesionales de aproximación a la audiencia. Palabra Clave, 20(1), 14-46. doi: https://doi.org/10.5294/pacla.2017.20.1.2

Patterson, T. E. (2007). Young People and News. Joan Sho renstein Center on the Press, Politics and Public Policy. Harvard University: John F. Kennedy School of Government.

Pérez Tornero, J. M.; Tejedor Calvo, S., Simelio Solà, N. y Marín Ochoa, B. E. (2015). Estudiantes universitarios ante los retos formativos de las Redes Sociales: el caso de Colombia. Estudios sobre el Mensaje Periodístico, 21(1), 509-521. Recuperado de https://revistas.ucm.es/index.php/ESMP/article/view/49108/45801.

Qayyum, M. A., Williamson, K., Liu, Y. H. \& Hider, P. (2010). Investigating the News Seeking Behavior of Young Adults. Australian Academic \& Research Libraries, 41(3), 178-191.

Rayén Condeza, R.; Bachmann, I. \& Mujica, C. (2014). El consumo de noticias de los adolescentes chilenos: Intereses, motivaciones y percepciones sobre la agenda informativa. Comunicar, 22(43), 55-64. doi: https://doi.org/10.3916/C43-2014-05

Said-Hung, E. M., Prati, R. C. \& Cancino-Borbón, A. (2017). La orientación ideológica de los mensajes publicados en Twitter durante el 24M en España. Palabra Clave, 20(1). doi: 10.5294/pacla.2017.20.1.10

Stanyer, J. (2012). Intimate politics: Publicity, privacy and personal lives of politicians in media saturated democracies. Cambridge, UK: Polity Press.

Tewksbury, D. \& Riles, J.M. (2015). Polarization as a function of citizen predispositions and exposure to news on the Internet. Journal Of Broadcasting \& Electronic Media, 59(3) 381-398. doi: https://doi.org/10.1080/08838151.2015.1054996

Valenzuela, S.; Arriagada, A. y Scherman, A. (2012). The Social Media Basis of Youth Protest Behavior: The Case of Chile. Journal of Communication,62(2), 299-314. doi: https://doi.org/10.1111/j.1460-2466.2012.01635.x 
BEATRIZ CATALINA-GARCÍA, MANUEL MONTES VOZMEDIANO Y ANTONIO GARCÍA JIMÉNEZ LOS JÓVENES UNIVERSITARIOS Y SUS PAUTAS DE CONSUMO Y DIFUSIÓN DE NOTICIAS SEGÚN...

Zubieta, E., Delfino, G. y Fernández, O. (2008). Dominancia social, valores y posicionamiento ideológico en jóvenes universitarios. Psicodebate, $8,151-170$.

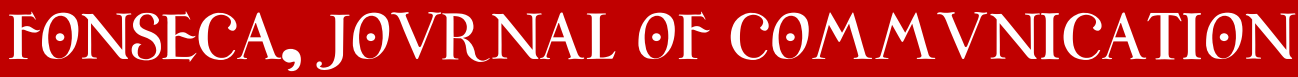

